\title{
Nonischemic ST segment elevation in hypertrophic cardiomyopathy due to chest wall deformity from kyphoscoliosis
}

This article was published in the following Dove Press journal:

International Medical Case Reports Journal

2I April 2010

Number of times this article has been viewed

\author{
Aleš Blinc' \\ Mirjam Gubenšek' \\ Mišo Šabovičl \\ Marko Grmek ${ }^{2}$ \\ Pavel Berden ${ }^{3}$ \\ 'Department of Vascular Diseases, \\ ${ }^{2}$ Department of Nuclear Medicine, \\ ${ }^{3}$ Department of Radiology, University \\ Medical Centre Ljubljana, Ljubljana, \\ Slovenia
}

Correspondence: Aleš Blinc Department of Vascular Diseases, University Medical Centre Ljubljana, Zaloška 7, 1525 Ljubljana, Slovenia Tel +386 I 5228032

$\mathrm{Fax}+386$ I 5228070

Email ales.blinc@kclj.si; ales.blinc@siol.net

\begin{abstract}
A 57-year-old male was admitted with suspected acute coronary syndrome. He reported experiencing moderate chest pain when walking during the day prior to admission, but had very prominent ST segment elevations in the precordial electrocardiography (EKG) leads. A physical examination revealed remarkable severe kyphoscoliosis with chest deformity. The patient's cardiac troponin levels remained normal, while cardiac ultrasound and magnetic resonance imaging of the chest confirmed hypertrophic cardiomyopathy (HCM) with severe thickening of the interventricular septum. Ischemic heart disease was ruled out by myocardial perfusion imaging with ${ }^{99 \mathrm{~m}} \mathrm{Tc}-\mathrm{MIBI}$ during rest and dipyridamole-induced stress without showing irreversible or reversible myocardial ischemia. Our diagnosis was that the chest pain was noncardiac in origin and that the pronounced ST segment elevations in the precordial EKG leads reflected the severely hypertrophic interventricular septum through the normally thick left ventricular free wall. The patient's chest wall deformity brought his septum and the ventricular free wall nearly parallel to the left side of the chest wall, allowing for complete expression of the reciprocal EKG pattern of septal hypertrophy. We suggest that EKG findings should always be interpreted with the chest wall shape being kept in mind.
\end{abstract}

Keywords: hypertrophic cardiomyopathy, EKG, ST segment elevation

When confronted with a patient who has prominent ST segment elevation in the electrocardiogram (EKG), a physician must quickly evaluate whether the patient has acute myocardial infarction (MI). Among the rare differential diagnoses, hypertrophic cardiomyopathy (HCM) should be considered. ${ }^{1-7} \mathrm{HCM}$ is a genetically-based myocardial disorder with heterogeneous clinical and morphological features. ${ }^{8-9}$ The diagnosis of HCM can be reliably established by echocardiographic demonstration of unexplained left ventricular hypertrophy, most often involving the interventricular septum. ${ }^{8}$ Conventional 12-lead EKG may be used for screening, since almost all patients with HCM have EKG abnormalities, most commonly in the form of abnormal Q waves, often accompanied by ST-T abnormalities and a pattern of left ventricular hypertrophy. ${ }^{10-11}$ In contrast, overt ST-segment elevation in anterior EKG leads is a rare finding in HCM which has been described in only a handful of cases. ${ }^{1-6}$

No explanation has been proposed for why ST segment elevation occurs only sporadically in patients with HCM. We report the case of a patient with HCM and severe chest deformity due to kyphoscoliosis who had pronounced ST elevation in precordial EKG leads that demonstrates how both the patient's cardiac abnormalities and shape of the chest influenced the 12-lead EKG. 


\section{Case report}

A 57-year-old Caucasian male was admitted with suspected acute coronary syndrome, primarily because of pronounced ST segment elevations in the precordial EKG leads (Figure 1). The day before admission he experienced two episodes of chest pain while walking. The pain was dull, at a maximum intensity 5/10 on the visual analogue scale, located to the left side of the sternum, and did not radiate elsewhere, but was accompanied by dyspnea and lightheadedness. On both occasions the pain subsided spontaneously after a few minutes of rest. The patient sought medical assistance the next day upon his wife's insistence, because he had reportedly suffered an anterior wall MI in 1987 and again in 1999, but had never been hospitalized in the acute phase. Both times, he completed an outpatient program of cardiac rehabilitation, and had good exercising capacity (10 MET) that allowed him to work up until a few years ago. He had a severe kyphoscoliotic deformation of the spine and chest due to an injury in childhood. Three years ago he had been diagnosed with syderopenic anemia because of persistent, low-grade blood loss due to gastroesophageal reflux disease, documented by endoscopy, and bleeding hemorrhoids. A recent colonoscopy had ruled out colon disease. His medication consisted of metoprolol $50 \mathrm{mg}$ twice a day, and oral iron supplements.
Upon admission he was not in acute distress. His blood pressure was $125 / 70 \mathrm{mmHg}$, with a respiratory rate of $24 / \mathrm{min}$, and a regular heart rate of $58 / \mathrm{min}$. His body height was $167 \mathrm{~cm}$ and body weight was $65 \mathrm{~kg}$. There was no congestion of the jugular veins and no peripheral edema. The thorax was severely kyphoscoliotic with a right-sided thoracic spine convexity (Figure 2), but lung sounds were normal. The apical impulse of the heart was not palpable because of the chest wall deformity. Heart sounds were of low intensity and a faint precordial systolic murmur radiating towards the axilla was heard. The abdomen was soft and peripheral pulses were palpable and symmetric.

The cardiac troponin I was normal both in the emergency room $(0.006 \mu \mathrm{g} / \mathrm{L})$ and 6 hours later $(0.008 \mu \mathrm{g} / \mathrm{L})$. Also, the $\mathrm{N}$-terminal-pro-B-type natriuretic peptide (412 ng/L) was normal. The patient's hemoglobin was $100 \mathrm{~g} / \mathrm{L}$ with a mean corpuscular volume of $77 \times 10^{-15} / \mathrm{L}$ and a mean corpuscular hemoglobin of $27 \times 10^{-12} / \mathrm{g}$. EKG results are shown in Figure 1 . In the patient's past medical records, deep precordial Q waves and ST segment elevation were noted in 1987 as having occurred, at which point he was told he had suffered a MI. An old EKG record was obtained from 1995 showing very similar findings as upon the current admission, thus ruling out dynamic EKG changes. There were no changes in the EKG during the

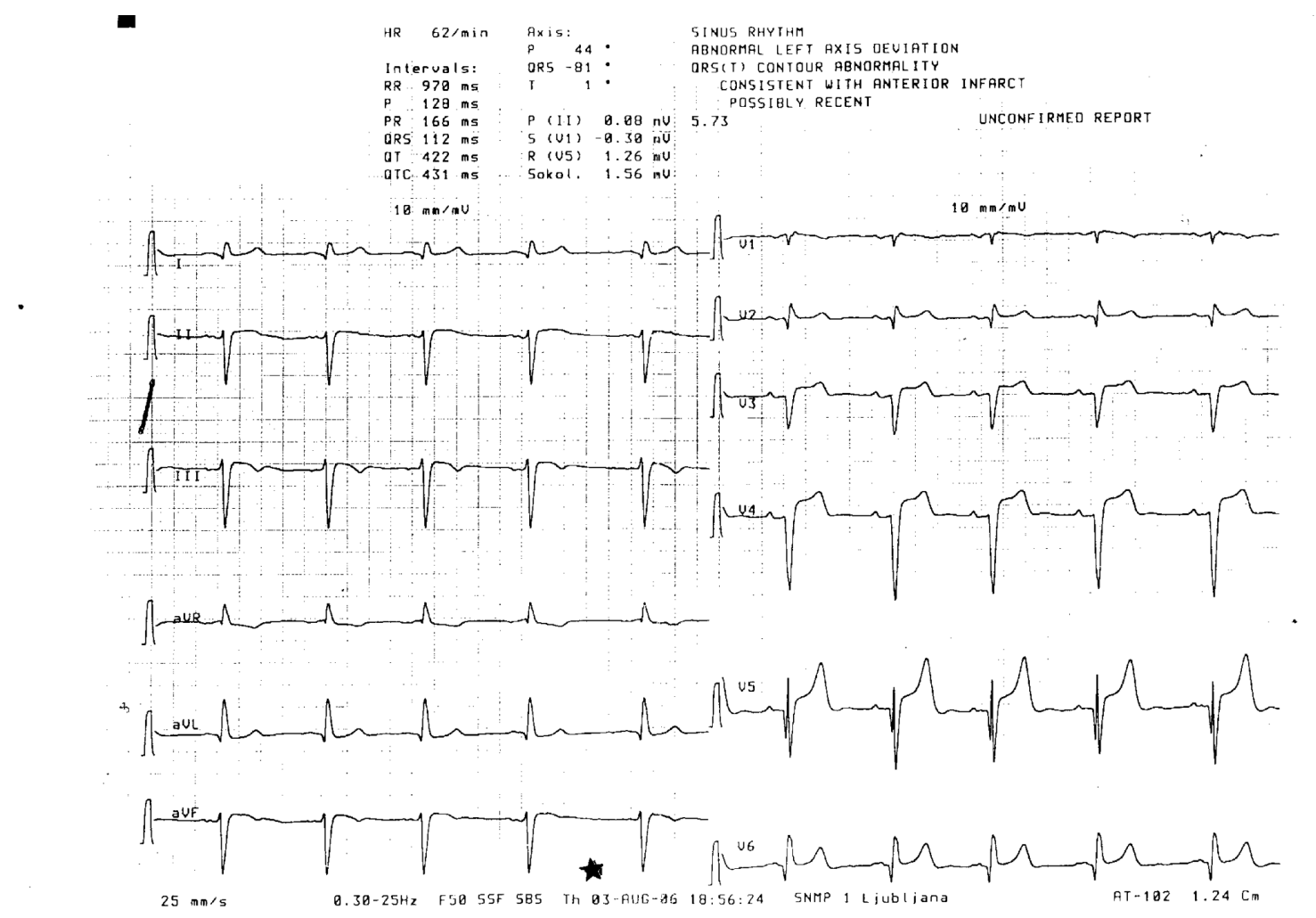

Figure I The EKG of our patient showed sinus rhythm of 62/min, an abnormal left axis deviation consistent with left anterior hemiblock, Q waves in the precordial leads, and prominent ST elevation in leads V3-V5. 


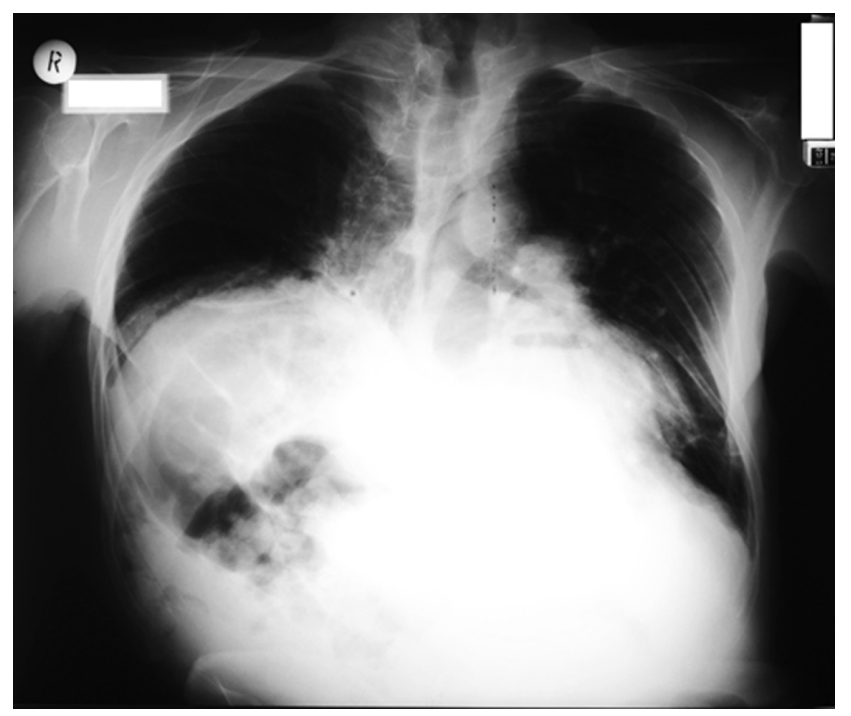

Figure 2 Chest $X$-ray of the patient with hypertrophic cardiomyopathy and kyphoscoliosis. Note the scoliotic curvature of the thoracic spine with the convexity on the right side and the high position of the right hemidiaphragm.

hospitalization and no rhythm disturbances were recorded. We performed a transthoracic, 2D cardiac ultrasound examination. There were no signs of impaired contraction of the anterior wall, but a severely thickened interventricular septum was found. The systolic function of the left ventricle was normal with an ejection fraction of 0.60 . A moderate mitral valve insufficiency was noted due to degenerative changes of the mitral valve leaflets, and only a mild transaortic gradient of up to
$25 \mathrm{mmHg}$ was noted. Magnetic resonance imaging of the heart (gradient echo, cine technique) confirmed that the patient had HCM (Figure 3). The thickness of the interventricular septum was $3.9 \mathrm{~cm}$ in systole and $3.5 \mathrm{~cm}$ in diastole. No significant dynamic outflow obstruction was found in the supine position, since the outflow tract remained at least $2 \mathrm{~cm}$ wide during systole. Myocardial perfusion scintigraphy with dipyridamoleinduced stress did not show reversible myocardial ischemia or irreversible ischemia, ie, myocardial scarring (Figure 4). No provocation tests with acetylcholine or hyperventilation were carried out to specifically rule out vasospastic angina, but the absence of EKG dynamics did not suggest that it was the cause of the patient's chest pain.

Immediately upon admission, while still under observation for suspected acute coronary syndrome, the patient received a transfusion of two units of packed red cells, raising his hemoglobin level to $116 \mathrm{~g} / \mathrm{L}$. Oral iron and a beta-blocker were continued, a proton-pump inhibitor (omeprazole) was started due to gastroesophageal reflux, and an angiotensin-converting enzyme inhibitor (perindopril) was prescribed because of mitral insufficiency. Although hypertrophic cardiomyopathy may cause chest pain and dyspnea, we had no proof of regional septal ischemia during dipyridamole-induced stress. Therefore we presumed the chest pain to be noncardiac in origin, while the accompanying dyspnea and lightheadedness was thought to be due to hyperventilation caused by anxiety. The patient

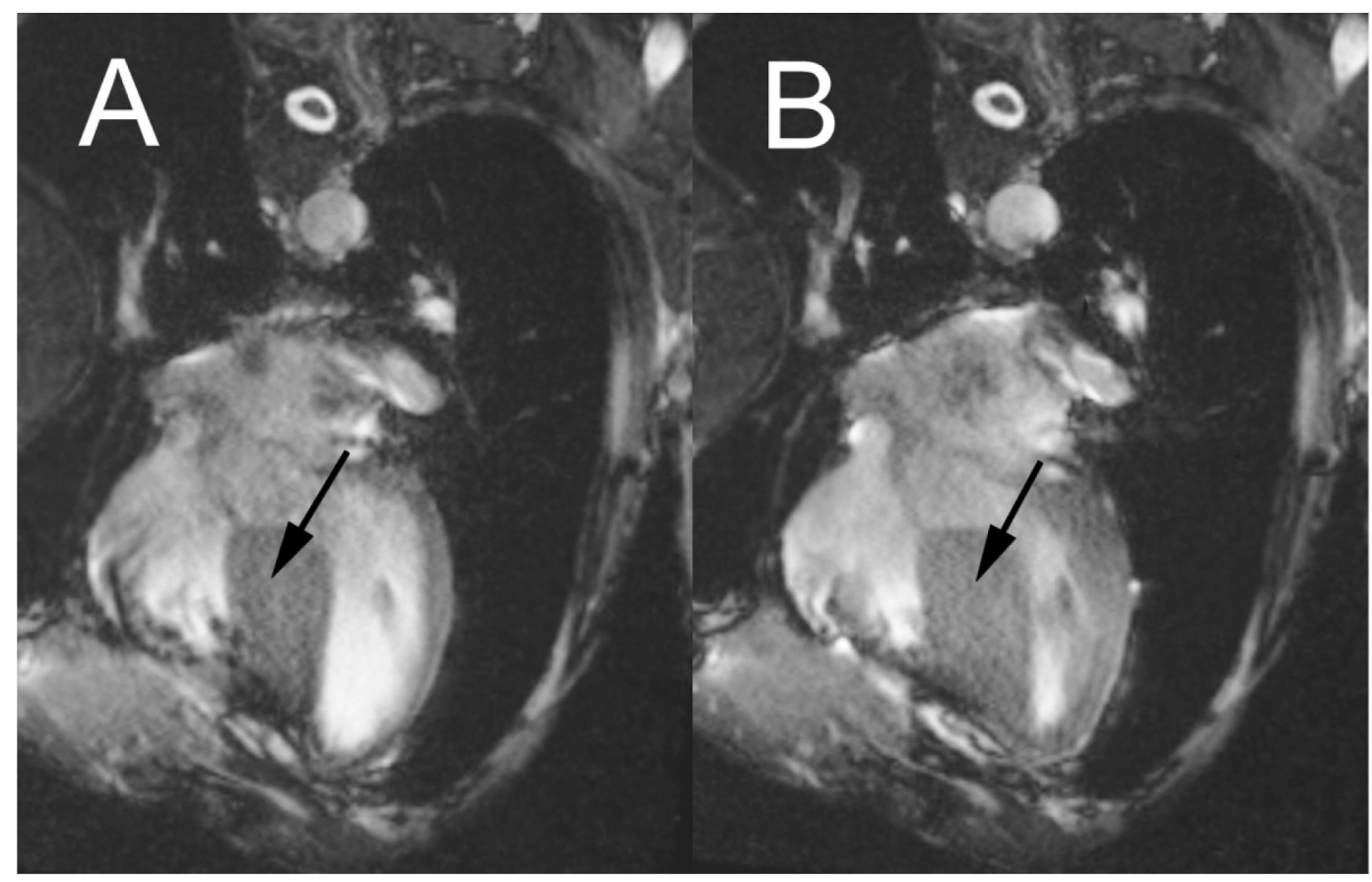

Figure 3 Magnetic resonance imaging of the heart in diastole A) and systole B) showing a severely hypertrophic interventricular septum (arrow) with a width of $3.5 \mathrm{~cm}$ in diastole and $3.9 \mathrm{~cm}$ in systole, a left ventricular-free wall of normal thickness, and a deformed chest wall with its left side running nearly parallel to the septum and free wall. 


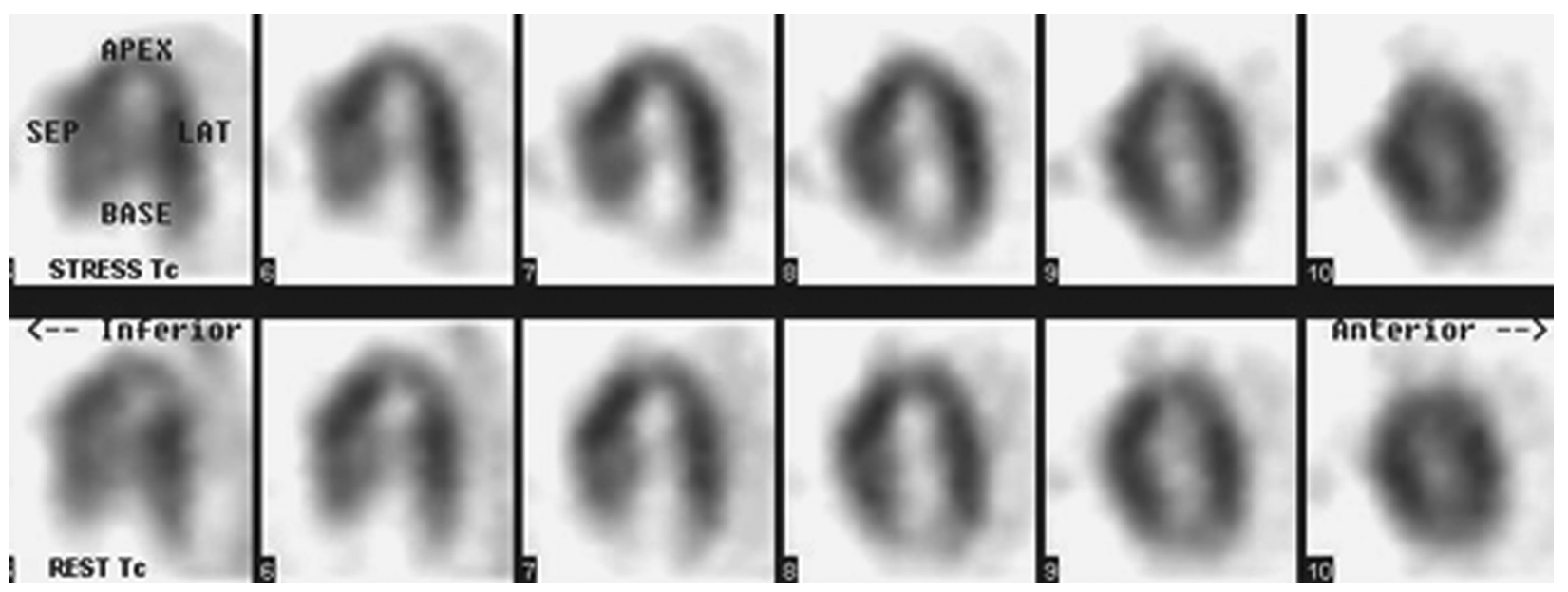

Figure 4 Myocardial perfusion imaging with ${ }^{99 \mathrm{~m} T c-M I B I}$ - long horizontal axis view. No signs of ischemia or scar during dipyridamole-induced stress were noted.

was discharged on the 5th day of hospitalization and scheduled for follow up by a cardiologist.

\section{Discussion}

Chest pain with anterior EKG lead ST segment elevation is a medical emergency. However, in addition to the possibility of acute anterior wall MI, the list of differential diagnoses is quite extensive, ${ }^{7}$ and should include HCM (Table 1). The hallmark of HCM is myocardial hypertrophy that is often asymmetric, and occurs in the absence of an obvious stimulus. ${ }^{8}$ Although any region of the left ventricle can be affected, hypertrophy frequently involves the interventricular septum, which may result in obstruction of the left ventricular outflow tract. ${ }^{8} \mathrm{HCM}$ is a genetically heterogeneous disease that may be inherited or may arise from a spontaneous mutation..$^{8-9}$ Over 300 dominant mutations in genes on several different chromosomes, encoding various components of the myocardial contractile system have been reported to cause HCM. ${ }^{9}$ Patients with HCM may be asymptomatic, or may present with symptoms such as dyspnea, presyncope/ syncope, chest pain, palpitations, orthopnea, paroxysmal nocturnal dyspnea, congestive heart failure, arrhythmias, or sudden cardiac death..$^{8-9}$

Abnormal Q waves are often associated with $\mathrm{HCM}^{10-11}$ especially in younger patients, where they have a $50 \%$ sensitivity and $>90 \%$ specificity for the diagnosis of HCM. ${ }^{10-11}$ Although ST and T wave abnormalities are often present, most often in the context of left ventricular hypertrophy, persistent ST segment elevation is a rare finding, described only in individual patients with $\mathrm{HCM} .{ }^{1-6}$

Table I Differential diagnosis of ST elevation in the precordial EKG leads, with specific reference to our case

\begin{tabular}{ll}
\hline Possible diagnosis & Ruled out/confirmed by \\
\hline Acute anterior wall myocardial infarction & Ruled out by serial troponin levels \\
Aneurysm of the anterior myocardial wall following myocardial infarction & Ruled out by cardiac ultrasound \\
Coronary vasospasm & Ruled out by absence of EKG dynamics \\
Acute pericarditis & Ruled out by absence of EKG dynamics, cardiac ultrasound and MRI \\
Reciprocal changes in VI andV2 reflecting hypertrophy of the left ventricle & Ruled out by normal left ventricular free wall thickness on cardiac \\
& ultrasound and MRI \\
„Early repolarization pattern« / »male pattern EKG« & Ruled out by establishing HCM and chest wall deformity as the \\
& likely cause of the EKG changes \\
Left bundle branch block & Ruled out by EKG \\
Pacemaker rhythm & Ruled out by EKG and absence of an external pacemaker \\
Preexcitation (Wolf-Parkinson-White) syndrome & Ruled out by EKG \\
Brugada syndrome & Ruled out by EKG \\
Intracerebral bleed & Ruled out by normal neurological status \\
Aortic dissection & Ruled out by MRI of the chest \\
Tension pneumothorax & Ruled out by normal lung sounds, chest X-ray and MRI of the chest \\
Hypertrophic cardiomyopathy & Confirmed by cardiac ultrasound and MRI of the chest
\end{tabular}

Abbreviations: EKG, electrocardiography; HCM, hypertrophic cardiomyopathy MRI, magnetic resonance imaging. 
A

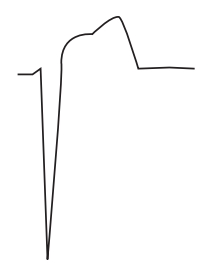

B

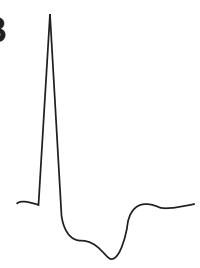

Figure 5 Our patient's EKG reading from lead V4 A) showed a classical ventricular hypertrophy pattern when inverted by $180^{\circ} \mathbf{B}$ ) which indicated that the anterior lead ST elevation was a reciprocal change reflecting septal hypertrophy. Abbreviation: EKG, electrocardiogram.

As late as 2000 , Welsh and Tymchak thought they were the first to describe persistent ST elevation in a man with HCM. ${ }^{4}$ In fact, the phenomenon of reciprocal changes in the precordial EKG leads, reflecting hypertrophy of the interventricular septum, was described by Braudo et al as early as $1964^{12}$ and was reviewed by Goldberger in 1979 in the context of explaining ST segment elevation, ${ }^{1}$ but no explanation has been given for why the finding is so rare. In our patient, the kyphoscoliotic deformation of the chest positioned the severely hypertrophied interventricular septum nearly parallel to the left ventricular free wall, which was of normal thickness, and the deformed left side of the chest wall (Figure 2), which allowed for maximal expression of the reciprocal EKG changes. Our patient's EKG reading from lead V4 showed a classical ventricular hypertrophy pattern when inverted by $180^{\circ}$ (Figure 5). The patient with HCM and anterior lead ST elevation, described by Welsh and Tymchak, was morbidly obese, ${ }^{4}$ which likely brought the heart into a horizontal position and also allowed for notable reciprocal EKG expression of the septal hypertrophy.

In conclusion, we hypothesize that ST segment elevation is most strongly manifested in those patients with $\mathrm{HCM}$ where the hypertrophied septum and left ventricular free wall, of normal thickness, are positioned in parallel with the left side of the chest wall, allowing for best reciprocal EKG expression of the septal hypertrophy in anterior leads. Thus, we believe EKG findings should be interpreted keeping in mind the chest wall shape of the patient.

\section{Disclosures}

The authors reports no conflicts of interest to this work.

\section{References}

1. Goldberger AL. Q wave T vector discordance in hypertrophic cardiomyopathy: septal hypertrophy and strain pattern. Br Heart J. 1979;42:201-204.

2. Kumar S. Persistent ST-segment elevation in hypertrophic subaortoc stenosis. Arch Intern Med. 1982;142:1957-1958.

3. Khan IA, Ajatta FO, Ansari AW. Persistent ST segment elevation: a new ECG finding in hypertrophic cardiomyopathy. Am J Emerg Med. 1999; 17:296-299.

4. Welsh RC, Tymchak WJ. An unusual case of ST elevation in a 39-year-old man. Can J Cardiol. 2000;16:215-217.

5. Luzza F, Carerj S, Oreto G. Hypertrophic cardiomyopathy with persistent ST segment elevation simulating acute myocardial infarction. Heart. 2004;90:380

6. Ha JW, Choi BW, Rim SJ, et al. Images in cardiovascular medicine. Extensive subepicardial fibrosis in a patient with apical HCM with persistent ST-segment elevation simulating MI. Circulation. 2005;112: e49-e50.

7. Sanders A, Froude A, Probst F. Do we intervene inappropriately for ST elevation? Emerg Med J. 2006;23:e10.

8. Elliott P, McKenna WJ. Hypertrophic cardiomyopathy. Lancet 2004;363:1881-1891.

9. Morita H, Seidman J, Seidman CE. Genetic causes of human heart failure. J Clin Invest. 2005;115:518-526.

10. Konno T, Shimizu M, Ino H, et al. Diagnostic value of abnormal Q waves for identification of preclinical carriers of hypertrophic cardiomyopathy based on a molecular genetic diagnosis. Eur Heart J. 2004;25:246-251

11. Dumont CA, Monserrat L, Soler R, et al. Interpretation of electrocardiographic abnormalities in hypertrophic cardiomyopathy with cardiac magnetic resonance. Eur Heart J. 2006;27:1725-1731.

12. Braudo M, Wigle D, Keith JD. A distinctive electrocardiogram in muscular subaortic stenosis due to ventricular septal hypertrophy. Am J Cardiol. 1964;14:599-607.
International Medical Case Reports Journal

\section{Publish your work in this journal}

The International Medical Case Reports Journal is an international, peer-reviewed open-access journal publishing original case reports from all medical specialties. Previously unpublished medical posters are also accepted relating to any area of clinical or preclinical science. Submissions should not normally exceed 2,000 words or

\section{Dovepress}

4 published pages including figures, diagrams and references. The manuscript management system is completely online and includes a very quick and fair peer-review system, which is all easy to use. Visit http://www.dovepress.com/testimonials.php to read real quotes from published authors. 